\title{
Impact of intraoperative fluid administration on associated outcomes in heac and neck cancer free flap surgery at a tertiary care hospital in LMIC
}

\author{
Waleed Bin Ghaffar, Faisal Shamim, Mujtaba Khalil, Fazal-ur-Rehman, Mubashir Ikram \\ Department of Anaesthesiology, Aga Khan University Hospital
}

\section{Introduction}

- Head and neck cancers are the sixth most common cancer; causing more than 350,000 deaths annually

- Pakistan falls geographically in a high-risk area for oral cancer.

- More challenging in Low and middle income countries due to limited resources

- Radical head and neck dissection with a free flap is an extensive procedure

- This procedure usually require large volumes of fluid.

- Anesthetic care has a fundamental role by regulation of hemodynamics and regional blood flow

- There is scant regional and no Local data

\section{Objective}

Primary Objective:

- To evaluate the impact of intraoperative fluid administration and its correlation with the postoperative medical and surgical complications in head and neck cancer free flap surgeries.

Secondary Objective:

- To identify demographic, preoperative and intraoperative factors predicting postoperative complications

\section{Material \& Methods}

\section{Study design}

- Retrospective observational study

\section{Inclusion Criteria}

- Elective head and neck cancer free flap procedures

- Age 18-65 years

- ASA I - III

\section{Exclusion Criteria}

- Patients with revision head and neck surgical procedures

- Patient receiving microvascular reconstruction as a salvage procedure for a previously performed free flap surgery

- Patients with history of head and neck cancer radiotherapy

\section{- Duration Of Study}

- 5 years

\section{Methodology}

- Approval from departmental research committee and hospital ethical review committee was obtained

- A retrospective review of all patients who underwent free flap procedures for head and neck cancer between January 2014 and December 2018 was conducted

- Medical records, including anaesthetic charts were reviewed

- A Performa was specifically designed for this study

\section{Data Analysis}

- All analyses were conducted by using the Statistical Package for Social Science version 19 (SPSS Inc., Chicago, IL)

- $P$ value of $\leq 0.05$ was considered statistically significant

\section{Results}

Demographic and clinical characteristics of patients $(n=224)$

\begin{tabular}{|l|c|}
\hline Variables & Point Estimate \\
\hline Age (Years) & $45.74 \pm 10.46$ \\
\hline Gender & [Range: $19-68]$ \\
Female & $32(14.5 \%)$ \\
Male & $192(85.7 \%)$ \\
\hline Co-morbid $\dagger$ & $68(30.4 \%)$ \\
Hypertension & $35(15.6 \%)$ \\
Dibetic Mellitus & $10.4 .5 \%)$ \\
IHD COPD & $9(4 \%)$ \\
Asthma & $14(6.3 \%)$ \\
Anemia & $14(6.3 \%)$ \\
Others & $8(3.6 \%)$ \\
\hline History of Addiction & $12(5.7 \%)$ \\
Smoking & \\
Betel Nut & $92(411 \%)$ \\
Others & $123(54.9 \%)$ \\
\hline Diagnosis & $26(11.6 \%)$ \\
Buccal Mucosa SCC & \\
Tongue SCC & $176(78.6 \%)$ \\
Lip SCC & $18(8 \%)$ \\
Mandible SCC & $3(1.3 \%)$ \\
Maxilla SCC & $10(4.5 \%)$ \\
Ameloblastoma & $4(1.8 \%)$ \\
Lower Alveolar SCC & $5(2.2 \%)$ \\
SCC Floor of Mouth & $7(3.1 \%)$ \\
& $1(0.4 \%)$ \\
\hline
\end{tabular}

Performed Procedure/ Intraoperative Fluid Information (n=224)

\begin{tabular}{|l|c|}
\hline \multicolumn{1}{|c|}{ Variables } & Point Estimate \\
\hline Procedure & \\
Fibular Flap & $83(78.6 \%)$ \\
ALTF & $104(46.4 \%)$ \\
Radial Forearm Flap & $22(9.8 \%)$ \\
Lateral Arm Flap & $15(6.7 \%)$ \\
\hline Duration of Anaesthesia (min) & $641[558-736]$ \\
\hline Duration of Surgery (Min) & $569[479-644]$ \\
\hline Length of hospital stay (days) & $5[4-5]$ \\
\hline Blood Loss (ml) & $697[500-1000]$ \\
& $\{$ Range: $60-2000\}$ \\
\hline Total Intraoperative Fluid (n=224) & $\mathbf{4 6 0 0 [ 3 5 2 5 - 5 5 0 0 ]}$ \\
\hline Total Crystalloid (ml) (n=224) & $4000[3500-5000]$ \\
\hline Ringer Lactate (n=209) & $4000[3000-4500]$ \\
\hline Normal Saline (n=71) & $1000[500-3000]$ \\
\hline Others: 5\% Dextrose (n=4) & $500[425-575]$ \\
\hline Total Colloid (ml) (n=145) & $\mathbf{6 0 0 [ 5 0 0 - 1 0 0 0 ]}$ \\
\hline Natural (n= 76) & $600[300-600]$ \\
\hline Packed Red Cell (n=77) & $600[300-600]$ \\
\hline Fresh Frozen Plasma (n=1) & $300^{*}$ \\
\hline Platelet (n=1) & $100^{*}$ \\
\hline Synthetic (n=121) & $500[500]$ \\
\hline Gelatin (n=115) & $500[500-500]$ \\
\hline Haemaccel (n=8) & $500[500-500]$ \\
\hline Albumin (n=0) & Nil \\
\hline
\end{tabular}

\begin{tabular}{|l|c|}
\hline Post-operative complications & Point Estimate \\
\hline Medical Complications & $70(31.25 \%)$ \\
\hline Pulmonary & $10(4.5 \%)$ \\
& \\
\hline Renal (Acute Kidney Injury) & $23(10.3 \%)$ \\
\hline Infectious (Sepsis) & $9(4 \%)$ \\
\hline Cardiovascular & $12(5.4 \%)$ \\
\hline Hematologic (Deep Venous Thrombosis) & 0 \\
\hline Unplanned mechanical ventilation / ICU admission & $9(4 \%)$ \\
\hline Others & $7(3.1 \%)$ \\
\hline & \\
\hline Surgical Complications & $18(8 \%)$ \\
\hline
\end{tabular}

Comparisons of characteristics of patients with and without Complications.

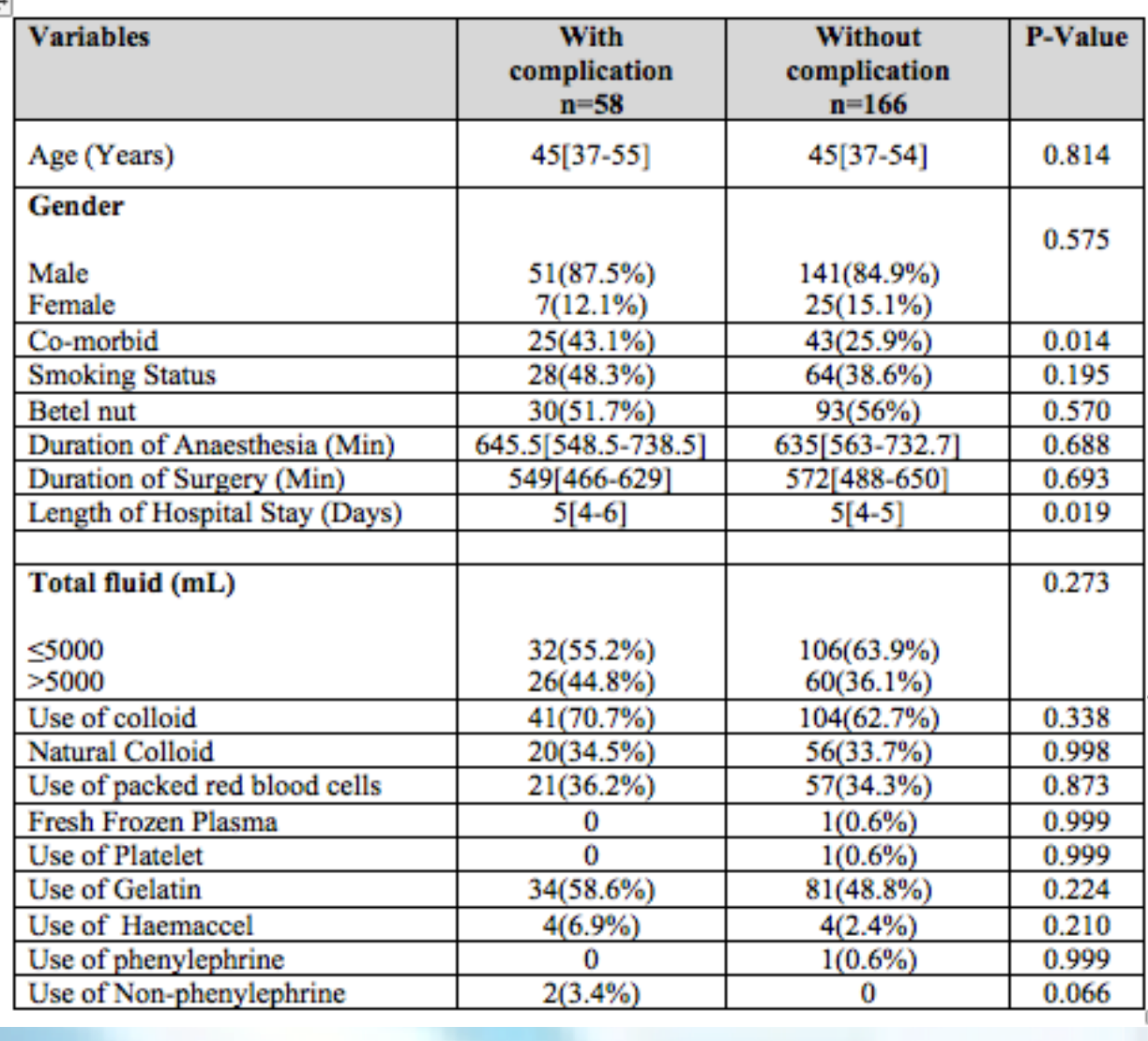

\begin{tabular}{|c|c|c|c|}
\hline Variables & $\begin{array}{c}\text { With } \\
\text { complication }\end{array}$ & $\begin{array}{c}\text { Without } \\
\text { complication }\end{array}$ & P-Value \\
\hline Total Fluid (ml) & $\begin{array}{c}n=58 \\
5000[3900-6075]\end{array}$ & $\begin{array}{c}n=166 \\
4550 \mid 3500-5500]\end{array}$ & 0.156 \\
\hline Total Crystalloid (ml) & $\begin{array}{c}n=58 \\
42503550-51251\end{array}$ & $\begin{array}{c}n=166 \\
40003500-50001\end{array}$ & 0.242 \\
\hline Ringer Lactate & $\begin{array}{c}4250505 \\
n=56 \\
4\end{array}$ & $\begin{array}{c}n=153 \\
n=0\end{array}$ & 0527 \\
\hline Normal Saline & $\begin{array}{c}4000[300-18000] \\
n=18\end{array}$ & $\begin{array}{c}40000=53 \\
n=500\end{array}$ & 0.780 \\
\hline Total Colloid (ml) & $\begin{array}{c}10000,50-27 \\
n-400] \\
800[500-1050]\end{array}$ & $\begin{array}{c}0000[50-304 \\
550[500-1000]\end{array}$ & 0.328 \\
\hline Natural Colloid & $\begin{array}{c}\mathrm{n}=20 \\
600[300-825]\end{array}$ & $\begin{array}{c}n=56 \\
600[300-600]\end{array}$ & 0.321 \\
\hline Packed Red Cell & $\begin{array}{c}n=21 \\
600[300-750]\end{array}$ & $\begin{array}{c}n=57 \\
600[300-600]\end{array}$ & 0.369 \\
\hline Synthetic & $\begin{array}{c}\mathrm{n}=35 \\
500[500-500]\end{array}$ & $\begin{array}{c}\mathrm{n}=86 \\
600[300-625]\end{array}$ & \\
\hline Gelatin & $\begin{array}{c}n=34 \\
500500-5001\end{array}$ & $\begin{array}{c}n=81 \\
500-500-5001\end{array}$ & 211 \\
\hline Haemaccel & $\begin{array}{c}n=4 \\
500350.8751 \\
5\end{array}$ & $\begin{array}{c}n=-4 \\
500-4500\end{array}$ & 0.999 \\
\hline
\end{tabular}

\section{Discussion}

- Till date, there has been no definitive consensus regarding optimal fluid protocols for patients undergoing $\mathrm{H}$ \& $\mathrm{N}$ free flap.

- Generally, complications occurs in H\&N free flap when fluid transfusion exceeds above $4000-5000 \mathrm{ml}$

- However in our study, more complications were noted in $<5000 m$ fluid group.

- AKI was the most common medical complication.

\section{Conclusion}

- We did not find statistically significant frequency of flap related complications associated with amount and type of intraoperative fluid administration in free flap $\mathrm{H} \& \mathrm{~N}$ cancer surgery

\section{Reference}

Characteristics and intraoperative treatments associated with head and neck free tissue transfer complications and failures. Otolaryngol Head Neck Surg 